\title{
Reply to the Correspondence by Untch et al. “Concerning Dediu M, Zielinski C: A Proposal to Redefine Pathologic Complete Remission as Endpoint following Neoadjuvant Chemotherapy in Early Breast Cancer" [Breast Care 2019; DOI 10.1159/000500624]
}

\author{
Mircea Dediu ${ }^{a}$ Christoph Zielinski ${ }^{b}$

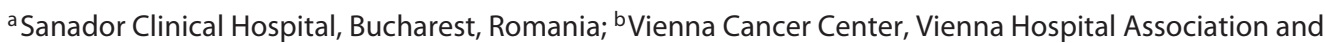 \\ Medical University Vienna, Vienna, Austria
}

We would like to thank Dr. Michael Untch [1] for his comments regarding our manuscript [2]. We want to strongly acknowledge that we are entirely in agreement with the benefits of using neoadjuvant therapy in breast cancer (NAT). These benefits have been extensively addressed and are very well summarized at the end of Dr. Untch's comment [1].

However, the key point of our considerations was to challenge the dogma of accepting pathologic complete remission (pCR) as a surrogate endpoint for overall survival (OS) in neoadjuvant clinical trials. Specifically, we evaluated the most pertinent data related to this issue and offered a reasonable explanation for the following paradox:

- Patients obtaining pCR upon NAT have a substantial better overall survival compared with their non-pCR counterparts (individual level).

- In randomized trials, a higher pCR rate induced by a certain regimen in one arm does not provide a survival advantage for the patients in this arm (trial level).

Related to the first part of the paradox, we came to the conclusion that the improved survival of patients with pCR is related to the prognostic significance of this event. We assumed that upon NAT, patients with good prognosis (i.e., small $\mathrm{T}$ and N0) are more likely transferred into the pCR category, while patients with poor prognosis (large T and node positive) are more likely transferred into the non-pCR category [2]. In the CTNeoBC meta- analysis, individual data were pooled and good and poor prognostic patients were mixed with each other [3]. The impact of NAT is restricted to its ability to "extract" the good prognosis individuals and to drive them into the pCR category. As such, the superior OS recorded for the pCR subcategory is directly correlated with the initial prognosis of patients, whereas NAT plays only a role as a driver for patient selection. The fact that patients achieving pCR enjoy a superior OS independent of the allocated NAT $[3,4]$ stands for the prognostic significance of pCR [5].

Coming to the second part of the paradox, we presented the data from large randomized trials confirmed by the meta-analyses and meta-regression, which showed no correlation of the magnitude of $p C R$ rate with OS [3, 6-9]. For instance, in the CTNeoBC meta-analysis, the authors found that the $R^{2}$ value for pCR as a surrogate for OS was extremely low $\left(r^{2}=0.24\right)$ and far below the accepted 0.75 threshold [10]. These figures make the surrogacy highly unlikely. The positive slope of the regression lines implies that treatments with a larger effect on pCR actually correlated with worse survival [3]. Moreover, various regimens able to provide a substantial increase in $\mathrm{PCR}$ rate in the neoadjuvant setting did not induce a clinically relevant benefit in the adjuvant setting [11-14]. Therefore, we came to the conclusion that PCR has no predictive significance related to NAT efficacy. 
We are aware that the difference between prognostic and predictive biomarkers might be challenging in modern oncology [15]. However, we want to put into question the rejection by Untch et al. of the prognostic quality of $\mathrm{pCR}$ based on the positive results of the post NAT trials $[16,17]$. They claimed that a prognostic significance of pCR "would be true only in the case that postneoadjuvant trials fail" [1]. In contrast to this, we consider that these trials have generated arguments supporting our theory: the post NAT trials included the non-pCR patients, which represent a highrisk patient subgroup extracted by NAT from the initially mixed population. However, this group harbors a poor prognosis from the outset. Therefore, it is permissive to conclude that the positive results of the post NAT trials are in line with our rationale by showing that poor prognosis patients selected by NAT are better served by intensified subsequent treatment. Thus, we would want to put into question that pCR might be "predictive for the further outcome of patients with non pCR" as claimed by Untch et al.

The paradox of $p C R$ in NAT trials was noted and reported extensively by others $[18,19]$ but no satisfactory explanation was given. Our perspective promotes the possibility that, individually taken, patients achieving pCR upon NAT will have a longer survival time due to their initial good prognosis. In the randomized trials, the regimens evaluated may have a different ability to translate the good prognosis patients into the pCR category hence, different pCR rates. Eventually, all patients got their primary tumor and lymph nodes removed by surgery, so their overall survival time will depend exclusively on the effect on the micrometastatic deposits. If the two regimens in the trial have similar efficacy in controlling the micrometastatic deposits, then no difference in survival will occur despite different $\mathrm{pCR}$ rates. Therefore, no correlation between pCR rate and OS improvement can be established, as noted in clinical trials and the metaanalysis. We do not exclude that a more intensive regimen may improve patients' outcomes, but this effect should be related to its ability to eradicate the micrometastases and not to the potential of inducing pCR.

Besides the above-mentioned considerations, we need to acknowledge that some biologic particularities may contribute to the $\mathrm{pCR}$ rate and its correlation with patient survival. For instance, the tumor infiltrating lymphocytes (TIL) ratio was correlated with pCR rate in neoadjuvant trials [20-23]. In a multivariate analysis, high-TIL status was an independent factor predicting favorable prognosis for triple-negative (TNBC) $(\mathrm{HR}=0.24, p=0.023)$ and HER2 positive breast cancer ( $\mathrm{HR}=0.13, p=0.036)$ [23]. Moreover, a significant inverse relationship between larger tumor burden (tumor size $p=0.01$; more nodes $p=$ 0.02 ) and the amount of TIL was noted, thus adding an additional argument in favor of the prognostic significance of pCR - here, in relationship with TIL status [24].
In TNBC, the BRCA mutational status significantly interferes with the pCR rate. For instance, in the GeparQuinto trial, a significantly higher pCR rate was associated with BRCA mutant versus BRCA wild-type tumors (50 vs. $31.5 \%$; $p=0.001$ ). However, $\mathrm{pCR}$ was a strong predictor of DFS only for patients without BRCA mutations $(\mathrm{HR}=0.18, p=0.004)$ despite the higher $\mathrm{pCR}$ rate in BRCA mutant patients [25].

All the data presented above argue in favor of pCR being related to multiple determinants (prognostic, immunologic, biologic) which independently can mediate the correlation between pCR and overall survival. Therefore, we consider that using pCR as a surrogate for NAT efficiency may be misleading and does not have "the potential to accelerate the progress in the medical treatment of breast cancer patients."

In conclusion, we want to strengthen our full agreement and support for the value of NAT in clinical practice. We just made a point in redefining the relevance of $\mathrm{pCR}$ in clinical trials. We strongly believe that the pCR surrogacy in breast cancer clinical trials should remain a matter of controversy.

\section{Disclosure Statement}

Dr. Dediu served as a consultant and received conference fees from Roche, Astellas, Accord, Boehringer Ingelheim, Amgen, Novartis, TEVA, Sandoz, Merck, Pfizer, Astra- Zeneca, BMS, Eli Lilly, Johnson and Johnson, MSD. Dr. Zielinski received consultancy fees and speakers' honoraria from Roche, Novartis, BMS, MSD, Imugene, Ariad, Pfizer, Merrimack, Merck KGaA, Fibrogen, AstraZeneca, Tesaro, Gilead, Servier, Shire, Eli Lilly, Athenex.

\section{Funding Sources}

None.

\section{Author Contributions}

All authors contributed equally.

References

1 Untch M, Loibl S, Fasching P. Concerning Dediu M, Zielinski A: "A proposal to redefine pathologic complete remission as endpoint following neoadjuvant chemotherapy in early breast cancer. Breast Care (Basel). 2019, DOI: 10.1159/000500624.

2 Dediu M, Zielinski C. A proposal to redefine pathologic complete remission as endpoint following neoadjuvant chemotherapy in early breast cancer. Breast Care (Basel). 2019, DOI: $10.1159 / 000500620$

3 Cortazar P, Zhang L, Untch M, Mehta K, Costantino JP, Wolmark N, et al. Pathological complete response and long-term clinical benefit in breast cancer: the CTNeoBC pooled analysis. Lancet. 2014 Jul;384(9938):164-72. 
4 Spring LM, Fell G, Arfe A, Trippa L, Greenup $\mathrm{R}$, Reynolds K, et al. Pathological complete response after neoadjuvant chemotherapy and impact on breast cancer recurrence and mortality, stratified by breast cancer subtypes and adjuvant chemotherapy usage: Individual patient-level meta-analyses of over 27,000 patients. 2018 San Antonio Breast Cancer Symposium. Abstract GS2-03. Presented December 5, 2018

5 Ballman KV. Biomarker: predictive or Prognostic? J Clin Oncol. 2015 Nov;33(33):396871.

6 Gianni L, Pienkowski T, Im YH, Tseng LM, Liu MC, Lluch A, et al. 5-year analysis of neoadjuvant pertuzumab and trastuzumab in patients with locally advanced, inflammatory, or early-stage HER2-positive breast cancer (NeoSphere): a multicentre, open-label, phase 2 randomised trial. Lancet Oncol. 2016 Jun; 17(6):791-800.

7 Sikov WM, Berry DA, Perou CM, Singh B, Cirrincione CT, Tolaney SM, et al. Abstract S2-05: Event-free and overall survival following neoadjuvant weekly paclitaxel and dosedense AC +/- carboplatin and/or bevacizumab in triple-negative breast cancer: Outcomes from CALGB 40603 (Alliance). 2015 San Antonio Breast Cancer Symposium. Presented December 9, 2015.

8 Untch M, Schneeweiss A, Salat C, et al. Longterm survival analysis of the randomized phase II trial investigating the addition of carboplatin to neoadjuvant therapy for triplenegative (TNBC) and HER2-positive early breast cancer (GeparSixto). Ann Oncol. 2017; 28(suppl_5):v43-v67.

9 Berruti A, Amoroso V, Gallo F, Bertaglia V, Simoncini E, Pedersini R, et al. Pathologic complete response as a potential surrogate for the clinical outcome in patients with breast cancer after neoadjuvant therapy: a meta-regression of 29 randomized prospective studies. J Clin Oncol. 2014 Dec;32(34):3883-91.
10 Buyse M, Molenberghs G, Burzykowski T, Renard D, Geys H. The validation of surrogate endpoints in meta-analyses of randomized experiments. Biostatistics. 2000 Mar; 1(1):49-67.

11 Baselga J, Bradbury I, Eidtmann H, Di Cosimo S, de Azambuja E, Aura C, et al.; NeoALTTO Study Team. Lapatinib with trastuzumab for HER2-positive early breast cancer (NeoALTTO): a randomised, open-label, multicentre, phase 3 trial. Lancet. 2012 Feb; 379(9816):633-40.

12 Gianni L, Pienkowski T, Im YH, Tseng LM, Liu MC, Lluch A, et al. 5-year analysis of neoadjuvant pertuzumab and trastuzumab in patients with locally advanced, inflammatory, or early-stage HER2-positive breast cancer (NeoSphere): a multicentre, open-label, phase 2 randomised trial. Lancet Oncol. 2016 Jun; 17(6):791-800.

13 Moreno-Aspitia A, McCormick Holmes E, Jackisch C, De Azambuja E, Boyle FM, Hillman DW, et al. Updated results from the phase III ALTTO trial (BIG 2-06; NCCTG (Alliance) N063D) comparing one year of anti-HER2 therapy with lapatinib alone (L), trastuzumab alone $(\mathrm{T})$, their sequence $(\mathrm{T} \rightarrow \mathrm{L})$ or their combination $(\mathrm{L}+\mathrm{T})$ in the adjuvant treatment of HER2-positive early breast cancer. J Clin Oncol. 2017 May;35:15_suppl, 502502.

14 von Minckwitz G, Procter M, de Azambuja E, Zardavas D, Benyunes M, Viale G, et al.; APHINITY Steering Committee and Investigators. Adjuvant Pertuzumab and Trastuzumab in Early HER2-Positive Breast Cancer. N Engl J Med. 2017 Jul;377(2):122-31.

15 Minden M. Is It Time to Redefine Prognostic and Predictive in Oncology? J Clin Oncol. 2016 May;34(14):1702-3.

16 Masuda N, Lee SJ, Ohtani S, Im YH, Lee ES, Yokota I, et al. Adjuvant Capecitabine for Breast Cancer after Preoperative Chemotherapy. N Engl J Med. 2017 Jun;376(22):2147-59.

17 von Minckwitz G, Huang CS, Mano MS, Loibl S, Mamounas EP, Untch M, et al.; KATHERINE Investigators. Trastuzumab Emtansine for Residual Invasive HER2-Positive Breast Cancer. N Engl J Med. 2019 Feb; 380(7):617-28.
18 Rose BS, Winer EP, Mamon HJ. Perils of the Pathologic Complete Response. J Clin Oncol. 2016 Nov;34(33):3959-62.

19 Vaidya JS, Massarut S, Vaidya HJ, Alexander EC, Richards T, Caris JA, et al. Rethinking neoadjuvant chemotherapy for breast cancer. BMJ. 2018 Jan;360:j5913.

20 Stanton SE, Adams S, Disis ML. Variation in the Incidence and Magnitude of Tumor-Infiltrating Lymphocytes in Breast Cancer Subtypes: A Systematic Review. JAMA Oncol. 2016 Oct;2(10):1354-60.

21 Denkert C, Loibl S, Noske A, Roller M, Müller BM, Komor M, et al. Tumor-associated lymphocytes as an independent predictor of response to neoadjuvant chemotherapy in breast cancer. J Clin Oncol. 2010 Jan;28(1): 105-13.

22 Ruan M, Tian T, Rao J, Xu X, Yu B, Yang W, et al. Predictive value of tumor-infiltrating lymphocytes to pathological complete response in neoadjuvant treated triple-negative breast cancers. Diagn Pathol. 2018 Aug; 13(1):66-77.

23 Asano Y, Kashiwagi S, Goto W, Takada K, Takahashi K, Hatano T, et al. Prediction of Treatment Response to Neoadjuvant Chemotherapy in Breast Cancer by Subtype Using Tumor-infiltrating Lymphocytes. Anticancer Res. 2018 Apr;38(4):2311-21.

24 Loi S, Drubay D, Adams S, Pruneri G, Francis PA, Lacroix-Triki M, et al. Tumor-Infiltrating Lymphocytes and Prognosis: A Pooled Individual Patient Analysis of Early-Stage TripleNegative Breast Cancers. J Clin Oncol. 2019 Mar;37(7):559-69.

25 Fasching PA, Loibl S, Hu C, Hart SN, Shimelis H, Moore R, et al. BRCA1/2 Mutations and Bevacizumab in the Neoadjuvant Treatment of Breast Cancer: Response and Prognosis Results in Patients With TripleNegative Breast Cancer From the GeparQuinto Study. J Clin Oncol. 2018 Aug; 36(22):2281-7. 